\title{
Computer Aided Design of a Crossing Current Resonant Converter (XCRC)
}

\author{
N.K.Poon* M.H.Pong** \\ * BSC Electronics Division, Hong Kong \\ ** Electrical \& Electronic Engineering Department, \\ Hong Kong University, Pokfulam Road, Hong Kong
}

\begin{abstract}
Computer aided design of the new Crossing Current Resonant Converter (XCRC) is introduced. The XCRC enables zero voltage switching without excessive voltage and current stresses. This makes the converter opernte with minimum dissipation. A systematic way of computer alded design of the XCRC is also introduced. Spreadsheet programme incorporates circuit equations for cileulation of component values. These values are then used for circuit simulation which further probes into the waveforms before the hardware is actually built. Finally the circuit is built and experimental results are included.
\end{abstract}

\section{INTRODUCTION}

Resonant converters are well-known for zero-voltage or zero-current operations which enable them to operate with low switching losses. However, because of the resonant waveforms involved, the switching devices are subjected to high voltage or current stresses. In some cases the overall dissipation in the switching device is increased. A new Crossing Current Resonant Converter (XCRC) is introduced which enables zero voltage switching without extra voltage or current stresses. This reduces device dissipation and enables operation without additional heat sinks, thus greatly reduces the size of the converter. Like all other converters, the XCRC switches between different states. In order to analysis and design the XCRC, a systematic computer aided design approach is used. Firstly, the circuit component values are calculated using a spreadsheet computer program which is widely available in personal computers. This gives a quick and inexpensive solution to carry out a preliminary design. The results so obtained are then applied to circuit simulation where waveforms can be simulated and checked. One can compare and examine the results calculated by the spreadsheet and those obtained from simulations and make adjustments as required. This process can be iterated until the designer is satisfied with the results. Then the designer can build the hardware with confidence.

This paper starts with a description of operation of the new Crossing Current Resonant Converter (XCRC). Analysis of the circuit then follows, the equations so derived are then implemented to a spreadsheet program. The results so obtained are used for circuit simulation. Finally a circuit is built and the waveforms obtained from the converter circuit is shown.

0-7803-1328-3/94\$03.00@ 1994 IEEE

\section{CtrcutT OPERATION OF THE XCRC}

The schematic diagram of the XCRC is shown in Figure 1. The circuit is similar to a half bridge topology but the circuit operation is quite different from conventional resonant circuits. The two capacitors $C_{1}$ and $C_{2}$ have small values and their voltages are allowed to swing up and down within the line voltage. Two diodes are connected in parallel with these capacitors. Cs1 and Cs2 are MOSFET drain-source capacitance which are inherent with the device.

By considering the current through the primary inductor $L$, we can identify three working states, namely the A) Power transfer state, B) Current circulating state and C) Crossover resonant state. By symmetric operation we shall consider a half cycle behaviour.

A) Power transfer state (Fig.2)

While $\mathrm{Ml}_{1}$ is turned on, current will flow through the transformer and charge up $\mathrm{C}_{2}$ until it reaches the supply voltage. Of course, $\mathrm{Cl}_{1}$ is being discharged at the same time. During this state, the transformer will "see" a voltage applied to it's terminals, so secondary voltage will build up and power will be transferred from the primary side to the secondary side and filtered by the output filter. This state will continue until the voltage across $C_{2}$ goes up to the supply voltage and turns on the diode $D_{1}$. Voltage across $C_{2}$ will be clamped to the supply voltage and force the circuit to go to the "Current circulating state". Throughout the design, continuous current through the output filter inductor is assumed.

\section{B) Current circulating state (Fig.3)}

While $\mathrm{Ml}$ is still on, the primary current will form a circulating current loop as illustrated in Fig. 3. The voltage across the transformer and the series inductor Lr will drop to approximately zero. In practice, the voltage across each component in the loop is nearly zero if the diode voltage drop is neglected. As a result, the di/dt in the loop is nearly zero and current circulates at a constant magnitude. This state ends when $M_{1}$ is turned off. This state controls the output power by varying its time period. This makes the $X C R C$ a frequency modulated converter. 

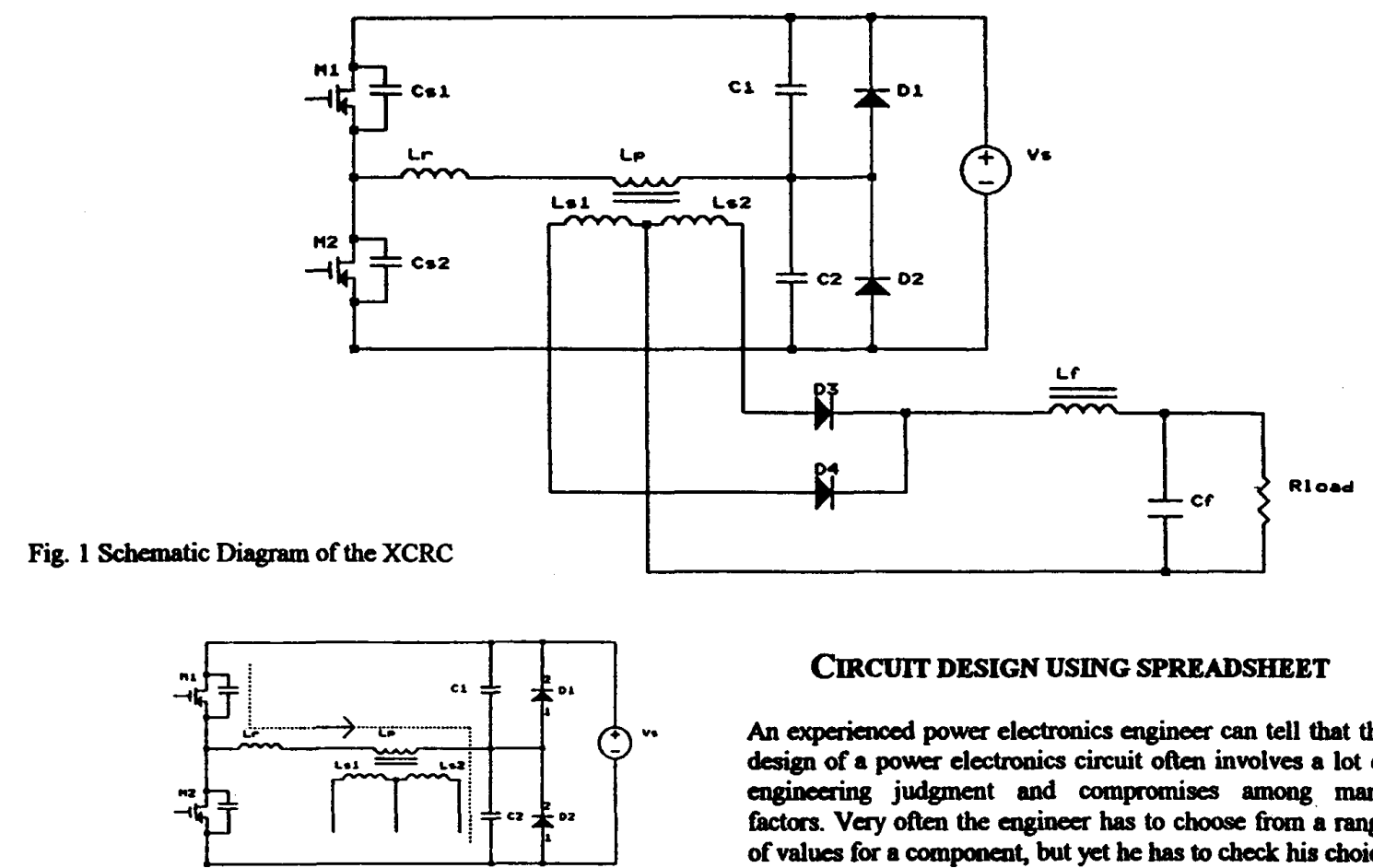

Fig. 2 Power Transfer State

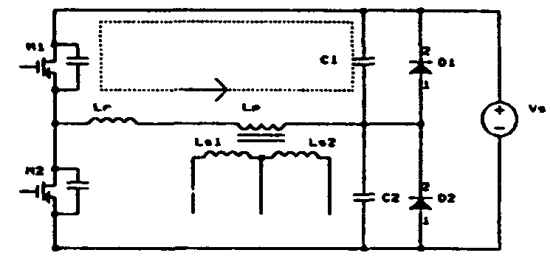

Fig. 3 Current Circulating State

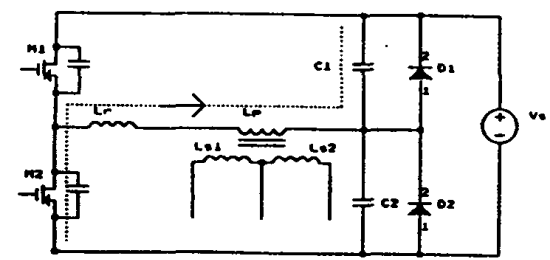

Fig. 4 Crossover Resonant State

\section{C) Crassover resonant state (Fig.4)}

At the beginning of this state, $M_{1}$ and $M_{2}$ are turned off to allow for a dead time in onder to avoid simultaneous conduction of $M_{1}$ and $M_{2}$. The primary current discharges $\mathrm{Cs}_{2}$ of $\mathrm{M}_{2}$ to $-0.7 \mathrm{~V}$ and current will be diverted through the body diode of MOSFET $M_{2}$ (which is not illustrated). While the body diode of $\mathrm{M}_{2}$ is conducting, it maintains a low drain source voltage of one diode forward drop. This facilitates the zero voltage condition for M2. M2 is then turned on which enables negative and positive current to go through the MOSFET. The operation cycle then repeats with the power transfer state. The Crossover resonant state is less straight forwand than the former two states. In fact there are three different modes in this state. More detailed descriptions can be found in the following section.

\section{CIRCUIT DESIGN USING SPREADSHRET}

An experienced power electronics engineer can tell that the design of a power electronics circuit often involves a lot of engineering judgment and compromises among many factors. Very often the engineer has to choose from a range of values for a component, but yet he has to check his choice against factors like operating voltage range, maximum and minimum load, capabilities of the controller, current or voltage stresses upon the power devices, etc. In onder to make the correct choice for the component concerned while keeping the overall converter performance in hand, a handy and responsive design tool is necessary and the spreadsheet program is a good candidate.

In this section the XCRC is analysed in detailed. The equations are applied to a spreadsheet program. Results are presented in graphical form. Simulated waveforms accompany the analytical equations.

The XCRC circuit to be analysed is shown in Fig. 5 . Throughout the analysis, continuous conduction is assumed and thus the filter and the load are represented by a constant current source. The waveforms concerned (simulated) are shown in Fig. 6 . The three operating states are indicated on the diagram.

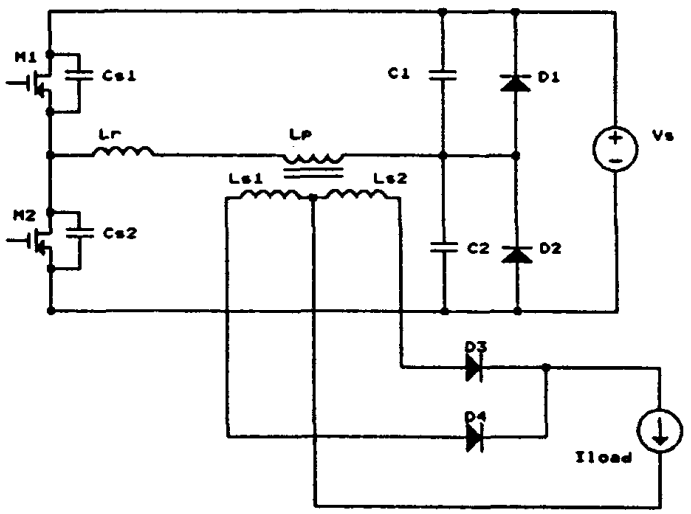

Fig. 5 Continuous operation mode 


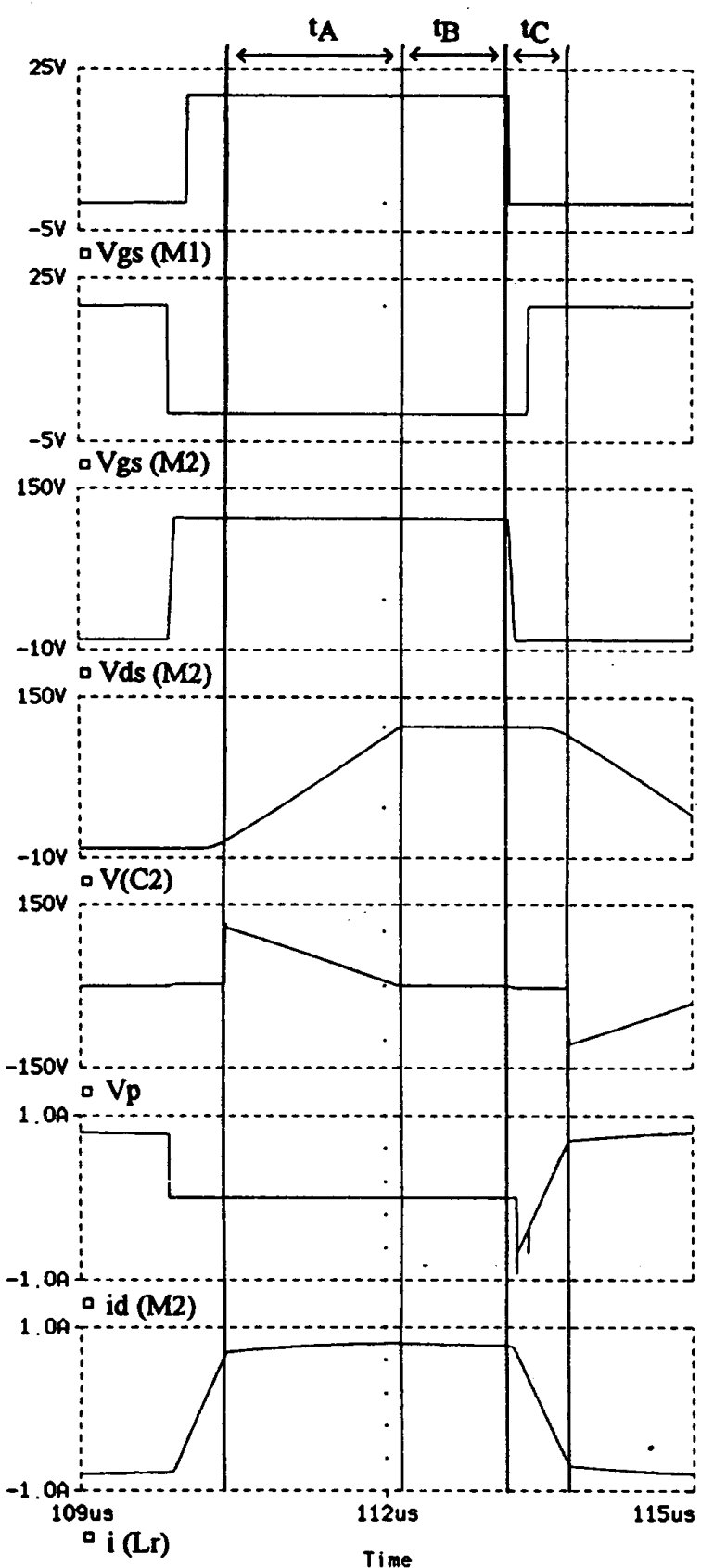

Fig. 6 The three operating states

\section{A) Power transfer state}

In this state the primary voltage across the transformer start at a certain voltage $V_{t} Z^{*}$ which is determined by the previous state. The amount of energy transferred by the transformer is represented by

$$
E=\int_{t_{z^{n}}}^{t} v_{p}+i_{p} t_{p} d t
$$

where $v_{p}$ is the transformer primary and ip the transformer primary current. As the secondary current is constant in the time period concerned, the primary current is also constant. The primary current charges up $\mathrm{C}_{2}$ and discharges $\mathrm{C}_{1}$. If $\mathrm{C}_{1}$ and $C_{2}$ have the same value and equal to $C$, the primary current is in fact charging up a total capacitance of $2 \mathrm{C}$. The capacitor voltage $V_{C 2}$ ramps up linearly in time $t_{A}$ where

$$
t_{A}=\frac{2 C \cdot\left(\operatorname{Vin}-V_{\left(z^{*}\right.}\right)}{i_{p}}
$$

where $V_{t} Z^{n}$ is the voltage across $C_{2}$ at the beginning of this state. It will be described in more detailed when the crossover resonant state is analysed. As the capacitance voltage ramps down linearly and ip is constant, the integral in equation (1) can be represented by the area under the triangular waveshape of $v_{p}$,

$$
\begin{aligned}
E & =\frac{1}{2} t_{A} \cdot\left(V i n-V_{t z^{\prime \prime}}\right) \cdot i_{p} \\
& =C \cdot\left(V i n-V_{t z^{\prime \prime}}\right)^{2}
\end{aligned}
$$

with a switching frequency of fs, the power transferred in the two symmetrical half cycle is

$$
P o=2 C .\left(V_{i n}-V_{i z^{*}}\right)^{2} . f s
$$

The output power is controlled by the switching frequency fs and the charging of the capacitors. It is obvious that because of this power transfer via the capacitors, output power is inherently limited by the switching frequency.

\section{B) Current circulating state}

Current now flows through one of the rectifier diode on the secondary side in this state. Again, current is assumed constant in the time period concerned. Variation of the time period t $B$ determines the overall switching frequency fs and regulates the output voltage.

\section{C) Crassover resonant state}

There are three modes in this state. The simulated waveforms are shown in Fig. 7. In the first mode ( $\left.\mathrm{t}_{\mathrm{z}}\right)$, the top transistor turns off. The drain source capacitance of the two transistors resonate with the inductor $\mathbf{L r}$, which brings the drain-source voltage of the lower transistor to zero. (Fig. 8) This facilitates the zero voltage turn on of the lower transistor.

In order to ensure that the drain-source voltage swings to zero, the following criteria must be met,

$$
\text { 2. } \operatorname{Vin}^{2} \cdot C \leq L r \cdot i_{p}^{2} \text {. }
$$

This defines the limiting value for the inductor $L r$. The time lapse $t_{z}$ for the Vds of the lower transistor to fall to zero is

$$
t_{z}=\frac{1}{\omega} \cdot \sin ^{-1}\left[\frac{2 V_{\text {in }} C \omega}{\text { ip }}\right]
$$

where

$\omega=\frac{1}{\sqrt{2 \mathrm{Lr} \cdot \mathrm{C}}}$ 


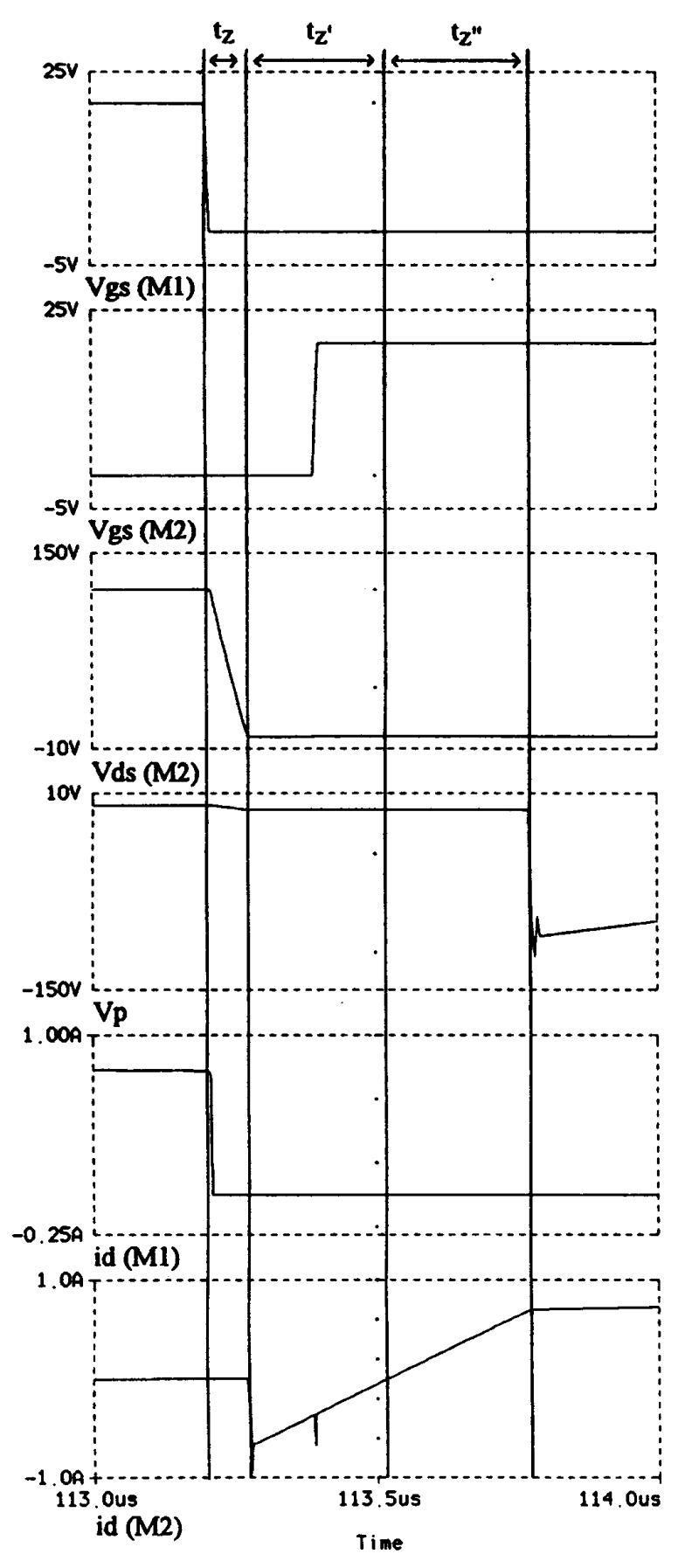

Fig. 7 The simulated Crossover resonant state

Once the Vds of the transistor reaches zero reverse current goes through the body diode of the transistor $\mathrm{M}_{2}$. In this mode the inductor Lr experiences the reverse voltage of Vin which reduces the current towards zero. The time lapse for this period is

$$
t_{z^{\prime}}=\frac{L r \cdot j_{t^{\prime}}}{V_{\text {in }}}
$$

where $i_{k^{\prime}}=i_{p} \cdot \cos \omega t_{z}$.
In order to schieve zero voltage switching, $\mathbf{M}_{2}$ must be switched on during the period $\mathrm{t}_{\mathrm{z}}$. As both $\mathrm{t}_{\mathrm{z}}$ and $\mathrm{t}_{\mathrm{z}}$ are functions of the primary current and voltage Vin, the instant at which $M_{2}$ switches on should be chosen with respect to the different operating conditions. Fig 8 shows that the selected turn on time of the transistor with respect to different values of $t_{2}$ and $t_{z}$.

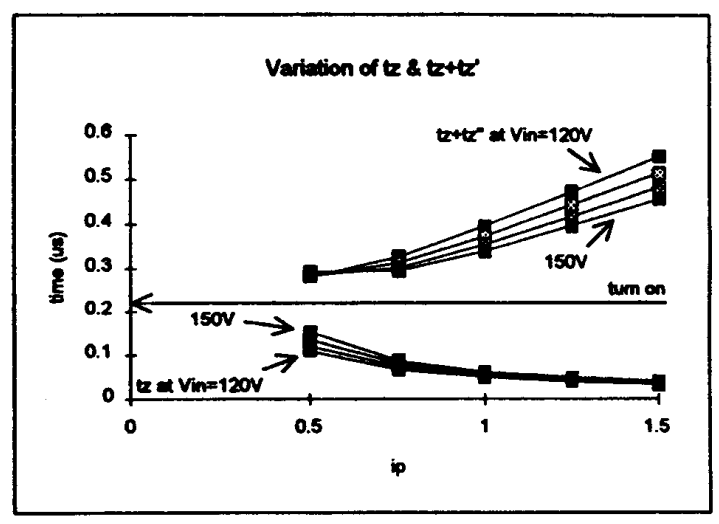

Fig. 8 Selection of transistor turn on time

The thind mode in this state is $Z^{\circ}$ during which positive current flows in the transistor. Now the inductor $\mathrm{Lr}$ and the two capacitors $C_{1}$ and $C_{2}$ form a $L C$ resonant circuit. The current swings up from zero until it takes up the corresponding secondary load current and stops the freewhoeling state on the secondary side. This mode ends with the reappearance of the transformer primary voltage. Hence the power transfer state described earlier comes in and the cycle continues.

The time $\mathrm{t}^{n}$ of this mode is the solution of a $\mathrm{L}$ and $2 \mathrm{C}$ resonant circuit with zero initial current and current ip at the end of the time period $z$

$$
t_{z^{*}}=\frac{1}{\omega} \sin ^{-1}\left[\frac{\text { ip.Lr. } \omega}{\operatorname{Vin}}\right]
$$

where $\omega=\frac{1}{\sqrt{2 \mathrm{LrCC}}}$

This time period $\mathrm{t}^{*}$ is important in the determination of the power transferred in each period. This is because $\mathrm{z}^{n}$ determines $V_{t}{ }^{*}$ in equation (2).

$$
V_{t 2^{\prime \prime}}=\frac{1}{2 C} \int_{0}^{t} \frac{V \text { in }}{L \cdot \omega} \sin \omega t
$$

It can be shown that

$$
V \text { in }-V_{t z^{\prime \prime}}=V \text { in. } \cos \omega t_{z^{\prime \prime}}
$$

and hence from equation (2)

$$
t_{A}=\frac{2 C}{\text { ip }} \cdot \operatorname{Vin} \cdot \cos \omega t_{z^{n}}
$$




\section{OPERATING LIMTSS AND TRANSFORMER DESIGN}

The presence of various time periods limit the operating frequency of the converter (Fig. 9). Assume that at the maximum frequency the time period $t_{B}$ in the current circulating state is set to zero for maximum power output. The maximum frequency is represented by

$$
f_{\max }=\frac{1}{t_{A}+t_{z}+t_{z^{\prime}}+t_{z^{\prime \prime}}}
$$

the variation of fmax is shown in Fig.10.

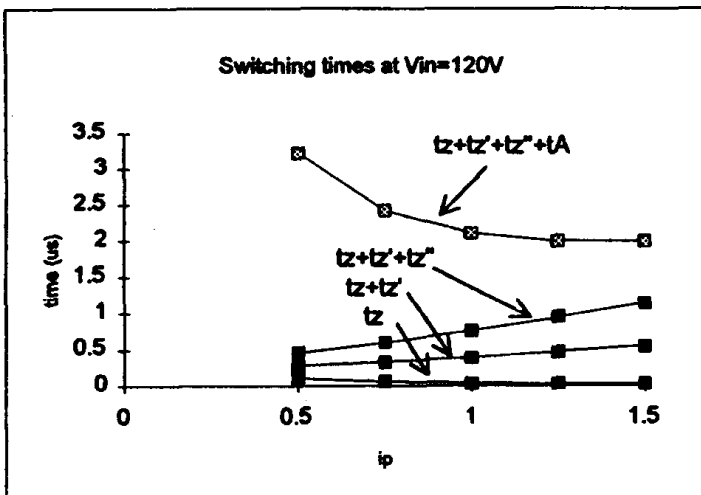

Fig. 9 Variation of $t_{z}, t^{\prime}, t_{z^{n}}$ and $t_{A}$

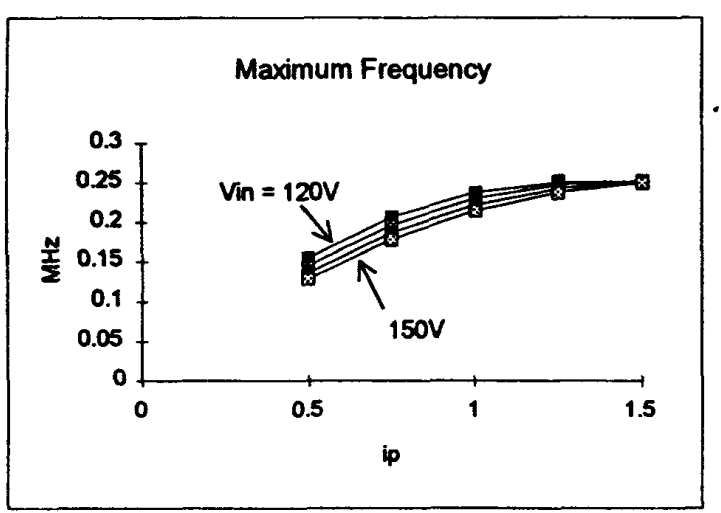

Fig. 10 Maximum Switching Frequency

The presence of maximum switching frequency imposes a limit on the power output which is shown in Fig.11. From the characteristic curves shown, this converter is most suitable for constant current application although it can also be used as constant voltage converter. The optimum operating primary current, ip, can be chosen which corresponds to the desired operating range.

Once ip is chosen, the transformer turns can be determined. The turns ratio makes sure that the primary current matches the output power,

$$
\frac{N_{p}}{N_{s}}=\frac{P_{0}}{\text { ip. } V_{0}}
$$

where Np and Ns are the primary and secondary turns respectively, Po is the output power and Vo the output voltage.

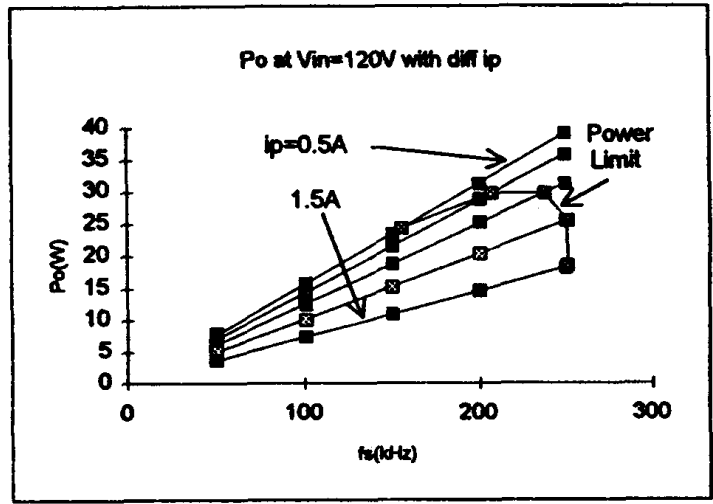

Fig. 11 Output power limit

Also, Np has to have a minimum no. of turns to avoid core saturation, by which

$$
\begin{aligned}
& N_{p} \cdot B_{\max } \cdot A_{e} \geq \frac{1}{2}\left(V i n-V_{t^{\prime \prime}}\right) \cdot t_{A} \\
& \text { and } N_{p} \geq \frac{C \cdot\left(V i n \cdot \cos \omega t_{z^{\prime \prime}}\right)^{2}}{i_{p} \cdot B_{\max } \cdot A_{e}}
\end{aligned}
$$

where $B_{\max }$ is the maximum flux density of the core, and Ae is the magnetic cross sectional area.

\section{EXPERIMENTAL RESULTS}

After confirmation of the design by SPICE circuit simulation, an off-line converter for $110 \mathrm{Vac}$ input is built. The output power of the converter is $20 \mathrm{~W}$ with voltage at $12 \mathrm{~V}$. Inductor Ir has an inductance of $41 \mathrm{uH}, \mathrm{Cl}_{1}$ and $\mathrm{C}_{2}$ are $5.8 \mathrm{nF}$, the transformer turns ratio is 1.7. The waveforms captured are shown in Fig. 12 which have been correctly predicted by simulations. More detailed waveforms showing the Crossover current state are shown in Fig.13. The MOSEFTs used are IRF830 with TO-220 package which can operate without any heat sink and the temperature rise is merely $8^{\circ} \mathrm{C}$ above ambient temperature. 


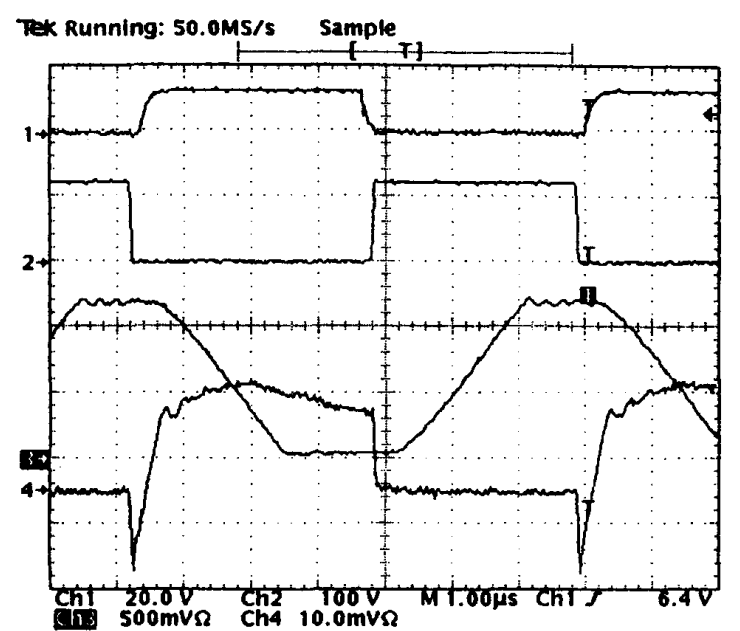

Ch1 : Vga(M2), 20VID

Ch3 : V(C2), 50V/D

Ch2 : Vds (M2), 100V/D

Ch4 : id(M2), 0.5AD

Fig. 12 Captured waveforms

TeK Running: 500MS/s ET Sample

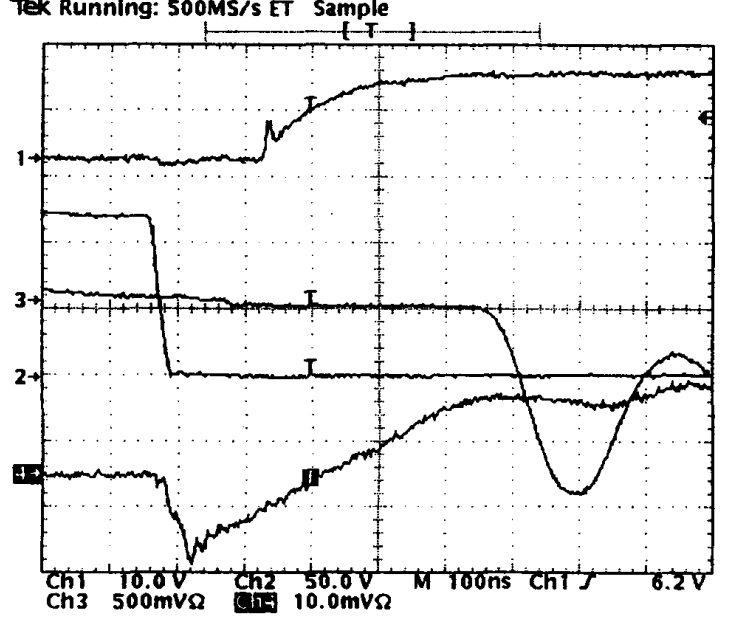

Ch1 : Vga(M2), 10V/D

Ch2 : Vds (M2), 50V/D

Ch3 : Vp, 50V/D

Ch4 : id(M2), 0.5A/D

Fig. 13 Waveforms at the Crossover current state

\section{Conclusions}

A novel Crossing Curnent Resonant Converter (XCRC) is introduced. This frequency modulated converter has the advantages of zero voltage switching, and minimum voltage and current stresses upon the switching devices. A systematic analysis and design approach is adopted. The circuit is analysed and the equations involved implemented to a spreadsheet program which is a versatile and low cost tool. Computed results are presented in graphical form and the operating points are chosen with respect to the operating limits. Design results are confirmed by simulations and an experimental XCRC is built. The experimental results confirm the outstanding features of the XCRC and the usefulness of the design approach.

\section{REFERENCES}

[1] M.H.Pong "Computer Aided Analysis of a Buck ZeroCurrent Switching Resonant Converter", Proceedings of the International Power Engineering Conference IPEC, pp.543-548, Singapore, March 1993.

[2] W.C.Ho, M.H.Pong "Power Loss and Efficiency Analysis of Quasi-Resonant Converters" International Conference on Industrial Electronics, Control and Instrumentation IECON 93, Hawaii, November 1993.

[3] A.H.Weinberg, L.Ghislanzoni "A New Zero Voltage and Zero Current Power-Switching Technique" IEEE Trans. on Power Eloctronics, Vol. 7, No.4, October 1992. 